\title{
EI lenguaje como elemento cultural de identidad social en la zona fronteriza del norte de México
}

\author{
Ma. Luisa Rodríguez Sala-Gómezgil \\ Instituto de Investigaciones Sociales \\ Universidad Nacional Autónoma de México
}

\section{Resumen}

Este ensayo sociolingüístico nos expone que en cualquiera de las formas en que se exprese el lenguaje, su función es eminentemente social y por lo tanto, ligada a lo cultural. El bilingüismo, para que tenga sentido social, deberá ser estudiado en relación con el contexto y mediante la distinción de varios dominios en los cuales es apropiado cierto comportamiento y tomando en cuenta, entre otros elementos: el ambiente familiar, de trabajo y de transacciones comerciales. Los diversos matices del bilingüismo están estrechamente vinculados a la interacción natural que implica vivir a lo largo de las fronteras entre comunidades lingüísticas diferentes. La importancia cultural se manifiesta en el uso evidente del propio grupo social que comparten el idioma común, en nuestro caso, el español.

Palabras clave: lenguaje, identidad social, zona fronteriza, bilingüismo, códigos culturales.

\begin{abstract}
This socio-linguistic essay states that, regardless any of the forms used to express a language, it has an eminently social function, for this reason it is also related to the cultural function. In order to have a social meaning, bilingualism should be studied in relation to the context and through the differentiation of diverse domains, where certain behavior is appropriate as well as other elements such as: family and work environments, and business transactions. The diverse shades of bilingualism keep a close relation to the natural interaction that means living by the borders amidst different linguistic communities. The importance of culture is manifested through the clear fact that the same social group shares a common language, in this case, Spanish language.
\end{abstract}

Keywords: language, social identity, border zones, bilingualism, cultural codes. 


\title{
EL LENGUAJE COMO ELEMENTO CULTURAL DE IDENTIDAD SOCIAL EN LA ZONA FRONTERIZA DEL NORTE DE MEXICO
}

\author{
Ma. Luisa Rodríguez Sala-Gómezgil \\ Investigadora titular del Instituto de Investigaciones Sociales UNAM \\ y actualnente investigadora visitante en el Centro de Estudios Fronterizos \\ del Norte de México (CEFNOMEX)
}

\section{LENGUAJE COMO ACTIVIDAD SOCIAL Y PRODUCTO NATURAL.}

Los aspectos sociológicos de la cultura han permitido captar la importancia que para su integración, su mantenimiento y su cambio, ejerce el aspecto simbólico, y más concretamente, el lenguaje mismo, como el representante por excelencia del mundo simbólico. Los sociólogos y lingüistas, tanto los clásicos como los contemporáneos, han visto el cimiento y la expresión de la cultura en el lenguaje, considerado éste en su dimensión totalizadora, que cubre tanto al lenguaje articulado como al gestual, al artístico y, en general, a todos aquellos que corresponden a la zona central: la simbólica designativa de la que hablan los lingüistas y que se contrapone y se complementa con la existencia de la zona periférica en la cual se ubican las formas expresivas y exclamativas, las cuales constituyen también parte de los códigos culturales.

En cualesquiera de las formas en que se exprese el lenguaje, su función -como ya ha sido establecida en especial por los sociolingüistas- es eminentemente social y por lo tanto íntimamente ligada a lo cultural. Esta afirmación merece un tratamiento más profundo, ya que conlleva en sí la justificación de parte del proyecto que sobre "Cultura de frontera e identidad nacional" se lleva a cabo en la zona fronteriza que corresponde al estado de Baja California. Para proceder a su delimitación partiremos especialmente de los trabajos que sobre el tema ha desarrollado nuestro colega Oscar Uribe Villegas, iniciador del estudio sociolingüístico en el ambiente académico no sólo mexicano sino también latinoamericano.

Es a partir de los trabajos de Humboldt que se inicia la conceptualización de la lengua como una actividad humana en su doble característica de actividad y producto del espíritu, dándole Vico su connotación cultural, Jespersen el de ser una actividad intencionada, Hermann Paul y los neogramáticos la concibieron como actividad psíquica y fue Whitney quien inició el enfoque social para su mayor formalización a cargo de Meillet, quien apoyado en las tesis de Durkheim vino a colocar a la lengua entre los fenómenos sociales. Sommerfelt recoge esta colocación y dice: "La lengua es un sistema de sig- 
nos que actúa como modelo colectivo independientemente del individuo particular. . . es un código externo al individuo, cuy as reglas tienen que ser observadas por éste" (Uribe Villegas, 0.1970). Es así como se establece que el lenguaje, basado en signos lingüísticos, según Granai no copia la cosa, sino que la sugiere o la indica, o sea, la simboliza, y al hacerlo así, la recrea: le da una existencia nueva y por lo tanto -seguimos a Uribe Villegas- la función simbólica somete a la comunicación a la arbitrariedad o al convencionalismo: la hace que dependa de un signo que se toma por lo que no es... Es necesario tomar a los signos lingüísticos por lo que no son, pero con la condición esencial de que la relación arbitraria que una la emisión vocal al concepto que significa, sea admitida por los interlocutores; que se dé un universo común de discurso y con ello que se pertenezca a una misma cultura, que se compartan pautas culturales similares y se tenga, a través de ellas, una visión del mundo "para nosotros". Se trata de un mundo para quienes hablamos uha lengua particular, y en consecuencia, de un mundo distinto al de los otros, al de quienes hablan otra lengua. Es así como el lenguaje marca pautas culturales diférentes y se constituye en sedimento de cultura. Cada lengua lo es de la cultura a la que pertenece en el pasado, pero en el presente contribuyen también a conformarla y a sentar las bases del desarrollo cultural futuro. La relación entre lengua y cultura se revela cuando el propio Jakobson hace descansar lo humano en un trípode, una de cuyas patas es el lenguaje, pero se trata ya de ese lenguaje que hará posible la comunicación entre miembros de un mismo grupo cultural y que dentro de ese grupo se muestra en su aspecto doble, en su capacidad de ser simultáneamente actividad y producto. Es actividad social y es producto cultural y esta afirmación, procedente de Uribe Villegas, lleva a considerar la estrecha relación entre lo social (actividad) y lo cultural (producto) que se aparta ligeramente de las afirmaciones de Humboldt y de Vico y se acepta que "el lenguaje está esencialmente vivo en el habla (actividad) y existe (aunque se encuentre cristalizado) y es actuante en la lengua (producto)". (Uribe Villegas, 0., 1970).

\section{LENGUAJE COMO REFLEJO Y MODELO DE LA REALIDAD SOCIAL Y CULTURAL.}

La relación entre ambas situaciones, la social y ia cultural, se entiende al considerar al lenguaje como una actividad social completa, una actividad que lleva en sí la relación dialéctica entre el regulador, limitador y coercionador y quien se somete o infringe las normas controladoras de cada cultura. En todo sistema cultural, sus prácticas, patrones y códigos están sujetos a un número corto o amplio de principios que se expresan a través del lenguaje, el cual simultáneamente, ejerce coerción sobre las ideas, las prácticas y los patrones de cada cultura. Es en esta relación dialéctica como el lenguaje refleja y modela al mundo, aunque hasta la fecha no haya una noción clara de cómo lo modela y el discernir acerca de este fenómeno constituye todo un reto filosófico. A pesar de esta limitante conceptual,es evidente que se da una estrecha relación entre el lenguaje y la expresión del mundo que nos rodea y de que los hechos lingüisticos revelan, junto con los otros aspectos culturales, 
no sólo los aspectos estructurales y objetivos de la sociedad sino también aquellos de carácter abstracto que constituyen la mundivisión en cada una de las etapas de su desarrollo histórico y cultural en las que se puede descubrir, a través del análisis lingüístico, las posiciones de equilibrio entre la forma externa de la lengua y la forma interior de la cultura.

En relación a esta temática consideramos necesaria la presentación e interpretación de aquellos planteamientos de Michel Foucault que, por su profundo acercamiento a lo que es el lenguaje en su aspecto cultural, constituyen una base teórica de interés substancial al enfoque que el proyecto sobre identidad cultural sustenta en lo que corresponde a la importancia del lenguaje en la determinación de los aspectos culturales, especialmente los relacionados con el conocimiento científico.

"Los códigos fundamentales de una cultura -los que rigen su lenguaje, sus esquemas perceptivos, sus cambios, sus técnicas, sus valores, la jerarquía de sus prácticas - fijan de antẹmano para cada hombre los órdenes empíricos con los cuales tendrá algo que ver y dentro de los que se reconocerá". (Faucault, M., 1978).

Foucault busca descubrir los códigos fundamentales de una cultura, pero concebida ésta en su expresión más amplia que se constituye en la representación de la suma de culturas particulares del mundo occidental. En su búsqueda de lo característico de cada etapa cultural, el autor indica uno de los rasgos propios de la cultura: su carácter dinámico y cambiante, la posibilidad de transformación de sus códigos. Pero, deberá entenderse este carácter dinámico en temporalidades muy amplias, a través de las cuales se producen transformaciones completas, que apenas son perceptibles en los límites generacionales, aunque en ciertos momentos cruciales del desarrollo histórico, los cambios son bruscos de una generación a la otra.

Son esos códigos los que permitirán que los hombres se reconozcan y se identifiquen, sea a través de su saber, a través de su experiencia; pero principalmente será a través de las formas de representación de esa saber y de esa experiencia.

De la obra de Foucault deberemos entresacar la importancia que reviste la relación que se le dé al lenguaje frente a las cosas y que será precisamente esa relación la que determinará los códigos de la cultura y de la sociedad correspondiente en una etapa dada.

Han sido las formas de representación a través del lugar que ocupa el lenguaje, las que han marcado los grandes cambios en el saber y con ello en los códigos culturales. La cultura occidental ha sufrido dos grandes vuelcos o dos grandes discontinuidades en el saber: la que se sitúa a mediados del siglo 
XVII, y que se corresponde con la primera gran revolución científica, y la que a principios del siglo XIX inicia la modernidad. Según Foucalt durante esos dos puntos cruciales del campo epistemológico occidental, se presentó un vuelco fundamental en la forma de ordenación y con ello de representación y por supuesto, del uso del lenguaje.

El cambio que trajo consigo el vuelco en el sistema epistemológico del mundo occidental, repercutió significativamente en los códigos culturales, con lo cual parecía poder establecerse una relación de causa a efecto entre episteme y cultura o más específicamente dicho, entre saber y códigos o expresiones culturales.

La tesis de Foucault que entre otros objetivos propugna por un humanismo cada vez más empeñado en poder descifrar auténticamente al ser humano, destaca para el estudioso de la ciencia y le permite ver, precisamente la gran influencia del conocimiento científico en el comportamiento de la sociedad en que se produce y el cómo esta sociedad, a través de las formas características de representación, hace palpable cada transformación epistemológica y determina una actitud. El cambio en el uso de las representaciones, no sólo las de orden lingüístico, sino aun las paralingüísticas y en general las que integran el ámbito semiológico, es manifiesto en los dos momentos culturales que analiza ampliamente el autor y que sirven de punto de partida para este intento de búsqueda de relación entre ciencia y análisis cultural.

Los dos puntos de inflexión en la episteme occidental han quedado ampliamente expuestos por Foucault y parecería no haber duda acerca de.las características en el cambio cultural que tales momentos trajeron consigo. Aunada a su concepción teórica, podría aventurarse la hipótesis de que se debería contemplar la aparición de un tercer vuelco a partir de lo que otro autor ha denominado la segunda gran revolución científica que se inicia con las grandes transformaciones que se han experimentado a partir de la tercera década del actual siglo.

No estamos en posibilidad de demostrar cómo ha repercutido este gran avance epistemológico en las formas de representación; sin embargo, no habrá duda alguna en los cambios tecnológicos y culturales que el desarrollo científico actual ha producido en los diferentes sistemas sociales. En algunas de las sociedades, en especial en las altamente tecnificadas, el impacto ha sido mucho más visible y manifiesto y ha conducido a un cambio cultural de consecuencias sociales aún no cabalmente vislumbradas, pero de efectos decisivos en el comportamiento y formas de expresión. Las sociedades en transición al industrialismo, vía la introducción de la avanzada tecnología, están aún en una etapa - por lo que se refiere a los cambios culturales- de preservación de sus códigos básicos. Pero a la vez, están con una enorme amenaza de sucumbir ante la embestida de las sociedades que ya han experimentado el nuevo vuelco epistemológico con sus consecuencias sociales. 
Este tercer punto de inflexión en la episteme occidental conduce -si acpetamos la hipótesis de Foucault - a una nueva relación entre las representaciones y las cosas en la cual el ser humano adquiere o bien una mayor dimensión, o bien se diluye en el avance científico y tecnológico, se ve arrastrado por éste y se pierde a sí mismo en una lucha desgastante por ocupar un lugar en cada sociedad y grupo específico. El impacto del avance científico y tecnológico presenta simultáneamente sus dos facetas: por un lado la positiva que contribuye a un mayor bienestar humano, por el otro, la cara negativa que trae consigo la posible disolución del ser humano, lo que ya Foucault insinúa en su texto, al afirmar: "el hombre ha de volver a esa inexistencia serena en la que lo mantuvo en otro tiempo la unidad imperiosa del discurso". Pero, hasta que esto se dé -si es que sucede así- el hombre pasa, ¿está pasando?, por una etapa de pérdida de su identidad, de cambio de códigos cultrales, de búsqueda de su sitio en la nueva dimensión que le presenta el cambiante aspecto de su sociedad, la que no acierta a digerir la acumulación de innovaciones científicas y tecnológicas, que, sin duda alguna, han introducido transformaciones fundamentales en cada uno de los códigos culturales y con ello han colocado al sistema social en una situación que lo imposibilita ya para guardar el equilibrio simbólico. Esta falla ocasiona, con mayor frecuencia de lo deseado, una deficiencia en el empleo de los símbolos que se traduce en una fundamental deficiencia en los procesos de interacción social.

\section{SOCIOLECTOS, BILINGUISMO Y PRESTAMOS: EXPRESIONES SOCIOLINGUISTICAS DE IDENTIDAD CULTURAL.}

Retomando lo lingüístico y poniéndolo en relación con lo social encontramos que, tanto Faoucault como Terracini complementados por Uribe Villegas, consideran que la forma interior del lenguaje se concecta con la de la cultura que expresa, y es así como podemos llegar a precisar la necesidad del análisis sociolingüístico en todo proyecto que pretenda conocer y determinar los patrones culturales y la identidad social. Es precisamente en la identidad social, en la identificación grupal, en la que el lenguaje desempeña un papel de suma importancia y de donde se puede desprender una hipótesis tentativa de trabajo a confirmar o descartar en la investigación que se realiza, que se enunciará así: En el grado en que se comparte el mismo lenguaje y el mismo sociolecto, en ese grado se puede hablar de identidad cultural similar; o bien: a mayor dispersión sociodialectal, mayor dispersión cultural, y, consecuentemente menor identidad social.

Dentro de toda comunidad hablante debemos reconocer un proceso de uniformización creciente que se suscita a lo largo del desarrollo cultural de cada comunidad o sociedad global. El proceso de aprendizaje lingüístico conlleva, en su aspecto social, la adquisición del lenguaje, que es individual: cada individuo aprende su idiolecto, pero lo tiene que aprender en la interacción social con otros hablantes y es a través de esa comunicación que se llega a una nueva interacción, con la que se establece entre los idiolectos; cuando 
éstos constituyen un conjunto de rasgos que les son comunes pasan a integrar el sociolecto que funciona dentro de una comunidad hablante homogénea. "Ese sociolecto está formado enteramente por aquellos rasgos que los hablantes reconocen como identificadores de una afiliación a una comunidad hablante diferente. El sociolecto está constituído por los rasgos que son iguales en los idiolectos. . . está basado en la observación de que, dentro de un grupo íntimamente trabado, aunque los hablantes noten las desviaciones del habla, las consideran sólo como idiosincráticas y no como marcadores grupales. Sólo cuando esas desviaciones son tales que identifican al hablante como miembro que pertenece a un grupo diferente podemos hablar de un nuevo sociolecto" (Haugen, E., 1974).

Son los sociolectos la expresión lingüística que la sociedad utiliza para señalar su diversificación y su estratificación y, a través del análisis de ellos es factible captar las mundivisiones propias de cada estrato social y señalar los rasgos culturales comunes y los rasgos diferenciales, que permitirán hablar del grado de identidad prevaleciente en la sociedad en estudio.

Interpretando a Haugen, podemos señalar que el problema en la determinación de las similitudes y diferencias sociolectales radica fundamentalmente en el hecho de que hay muchas clases de sociolectos y que ninguno es homogéneo, por lo cual resulta indispensable determinar unidades y criterios que permitan distinguir un sociolecto de otro y "grados de semejanza que permitan combinar objetivamente diferentes sociolectos dentro de una misma clase" (Haugen, E., 1974) y en cada sociolecto característico y ya establecido deberemos considerar, a su vez, la presencia de diferentes registros, o subdialectos. Son estas unidades las que están más estrechamente vinculadas a lo social, pero son también las que en el ámbito de la identidad pueden llevarnos a una diversificación tal que haga difícil la localización de los niveles buscados.

Tanto el uso de sociolectos como de registros implica la pertenencia a un grupo específico y al desempeño dentro de ese grupo de papeles sociales; por lo general el empleo de subdialectos y registros, más que identificar al grupo social del hablante, vinculan a éste al contexto en el cual está hablando y por lo tanto, interactuando, "el registro corresponde a los papeles de hablante y de interlocutor, al ambiente, al tópico y al medio". Los sociolingüistas hacen observar que el uso apropiado de los registros forma parte del aprendizaje de la propia lengua" (Haugen, E., 1974). Los sociólogos deberemos añadir que además, forma parte del proceso de socialización que señala a cada hablante qué registro utilizar de acuerdo al contexto en que se desempeñe y de acuerdo al papel que en ese contexto y situación le corresponda desempeñar. A través del estudio de los registros, algunos autores contemporáneos (Ure y Ellis, 1974), han logrado precisar: la forma en que cada situación social modela el lenguaje, la manera en que esa modelación del len- 
guaje hace que los participantes de la situación tomen conciencia de ella, y el modo en que la sociedad cotrola tanto la situación como a los participantes.

La multiplicidad de códigos es parte del sistema sociolingüístico de una comunidad, que como ya vimos puede, además, variar a través de su desarrollo cultural el empleo de diferentes códigos, sean sociolectos, sean registros, se extiende, ya fuera de los límites de una lengua, al fenómeno del bilingüismo y de los préstamos de otras lenguas.

Serán estas dos formas de comportameinto lingüístico a las que demos especial atención en el estudio de la identidad cultural en una zona fronteriza, ya que ellas denotan, en especial, la interacción sociocultural a lo largo de las fronteras entre comunidades de diferente procedencia lingüística.

El bilingüismo, para que tenga significado social, deberá ser estudiado, como propone Fishman, en relación con el contexto y mediante la distinción de varios dominios en los cuales es apropiado cierto comportamiento y entre los cuales se podrán considerar - para los fines específicos del proyecto -el ambiente familiar, el ambiente de trabajo y el ambiente de transacciones comerciales; en ellos hay que compartir del hecho de que "a las relaciones sociales hay que verlas en términos de redes de comunicación dentro de las cuales los individuos desempeñan varios papeles en varias situaciones" (Haugen, E., 1974. En cl caso de las comunidades fronterizas el bilingüismo presenta una especial connotación social y cultural y está implicando la existencia de lo que se conoce como "lealtad social plural del bilingüe" y que refleja una lealtad más amplia; la que se da frente al grupo y frente a su cultura, y que se vuelve consciente sólo cuando el grupo se ve amenazado (como sucede en el caso de los chicanos y otros grupos hispanoparlantes radicados en comunidades anglosajonas). Entre los individuos que viven en la zona fronteriza el análisis de su bilingüismo deberá ser considerado en una definición amplia, como la que propone Haugen, y que "incluiría virtualmente a todo el que tuviera que aprender y obtener aunque sólo fuera un barniz de una segunda lengua (Haugen, E., 1974), el inglés en este caso, cuyo aprendizaje y uso va a variar en grado de acuerdo al papel o papeles sociales que los individuos desempeñan en esa sociedad en los diferentes dominios sociales. Es muy probable que el ama de casa, en general, emplee rudimentaria o ampliamente la lengua extranjera en el dominio de las transacciones comerciales que se efectúan en las tiendas norteamericanas localizadas en las zonas menos cercanas a la frontera. Es también probable que el prestador de servicios, el comerciante y el profesionista hagan uso de un bilingüismo parcial o total en sus relaciones laborales y comerciales con los turistas que visitan las localidades mexicanas en busca de servicios o de adquisición de bienes materiales; algo similar sucedería con quienes se trasladan a trabajar en las comunidades norteamericanas en donde el uso del inglés estará determinado por el tipo de trabajo que se desempeñe y variará, desde un empleo rudimentario -en las actividades de servicios domésticos- hasta un dominio total en el ejercicio de una profesión o un oficio calificado. 
Estos diversos matices del bilingüismo están estrechamente vinculados a la interacción natural cara a cara que implica el vivir a lo largo de las fronteras entre comunidades lingüísticas diferentes, y si bien tiene su importancia cultural, ésta se manifiesta en forma evidente en el uso de la lengua extranjera en la interacción con los miembros del propio grupo social que comparten el idioma común, en nucstro caso, el español. Aquí estamos frente a la otra modalidad de la multiplicidad de códigos o registros, la que hemos denominado: préstamos de otras lenguas.

El fenómeno que implica la inclusión de préstamos lingüísticos y de registros extranjeros, con o sin una adaptación fonética y gráfica, parecería estar indicando cl grado de penetración cultural y la propensión a un alejamiento de la identidad cultural.

Los préstamos de otras lenguas constituyen uno de los elementos innovadores de las lenguas y como tales tienden a modificar el uso lingüístico y con ellos algunos de los patrones culturales de acuerdo a la incidencia de esos préstamos. Según Ottalengo y Paulín, esos préstamos se incorporan sin alterar la forma que tenían en el idioma de origen o pueden sufrir una adaptación de uno de los siguientes tipos: 1) las formas originales se adaptan tanto al sistema fonético como al gráfico de la lengua que recibe el préstamo;2) la forma original sólo se adapta gráficamente; y 3) la forma original sólo se adapta fonéticamente. Las deformaciones fonéticas y gráficas de los préstamos introducen en el idioma que los adopta acepciones distintas y contenidos diferentes a los originales, y esto hace que -después- se dificulte el intercambio entre las dos sociedades y las dos culturas. Con el tiempo, este intercambio se dificulta aún más, porque la cvolución de los términos tomados en préstamo no sigue en los idiomas prestatarios vías paralelas a las de su evolución en los idiomas prestamistas.

Los factores sociolingü isticos que propician la menor u mayor inclusión de los préstamos y le dan a cad a uno modalidades propias son: a) la diferencia y contacto de las sociedades en las que se hablan las lenguas respectivas; b) la superioridad técnica de la sociedad que presta sobre la sociedad que recibe; c) la diversidad de recursos lingüísticos de cada lengua; d) la habilidad de los usuarios para manejar lingü ísticamente esos recursos; y, e) la decisión o falta de decisión de esos hablantes para utilizar hasta el máximo los recursos lingüísticos de su propia lengua. Muchos de los préstamos, en especial los que se incorporan sin modificación alguna, se explican precisamente por una actitud psicosocial de los individuos que denota una situación de predominio cultural de la sociedad de donde provienen los términos y de su aceptación tácita, por comodidad y por pereza para la búsqueda del término adecuado en el propio idioma. En esta introducción de préstamos, los factores de política sociocultural tienen una especial importancia, pues serán ellos los que faciliten la vía de entrada o bien los que puedan frenar su incorporación indiscriminada. Las vías más transitables para los préstamos son las que ofrecen los 
macrodifusores: prensa, radio, televisión; los cuales, con la aceptación y uso de términos extranjeros propician su empleo por los lectores, radioescuchas y televidentes. El éxito que tengan estas vías en la introducción de préstamos dependerá de la política cultural que cl Estado desarrolle y del control que exista para que esa política se cumpla, en el supuesto caso de que fuera una política proteccionista de la cultura nacional y que, dentro de la concepción de cultura, se diera especial atención a la conservación y salvaguarda del idioma nacional u oficial.

En términos generales, y siguiendo a Uribe Villegas, podemos decir que la imposición de una lengua sobre otra, el desplazamiento de una lengua hacia otra y la aceptación de préstamos de una lengua extranjera se producen cuando se dan en la lengua extranjera una o varias de las características siguientes: "cuando sus hablantes o son numerosos o son poderosos o numerosos y poderosos; cuando se mezclan libremente con los hablantes de la otra lengua; cuando su idioma es portador de una cultura prestigiosa y de una tecnología avanzada; cuando sirve de vehículo para formas superiores de administración y es empleado por quienes tienen voluntad de incorporar a determinada población dentro de los límites jurisdiccionales de su Estado, o cuando quienen lo hablan tratan de propagar, por su medio, entre las poblacion alienígena, determinadas creencias y prácticas religiosas" (Uribe Villegas, $\mathrm{O}$. 1970). Y añadiríamos nosotros, cuando la política del Estado es de tendencia imperialista y a través de la lengua se busca influir en los patrones culturales y económicos.

La imposición, el desplazamiento y la introducción de préstamos no se logra cuando se dan una o varias condiciones físicas, sociales o culturales como pueden ser: cuando el grupo hablante puede aislarse en zonas periféricas o marginales de difícil acceso; cuando se vincula la lengua propia con una forma cultural o civilizatoria que se considera preferible aún por encima de la de los administradores o de los hablantes que pertenecen al grupo dominante económicamente; cuando la política cultural del Estado promueve una fuerte identificación nacional que se basa, entre otros factores, en la conservación del lenguaje como la institución que les proporciona a los miembros de una sociedad el sentido más fuerte de su identidad, y en torno a la cual se configuran todos los restantes símbolos de identificación nacionales.

Cuando no existen condiciones favorables para una fusión total o parcial se producen situaciones de bilingüismo más o menos intenso, según sea el número de características favorables para el desplazamiento, la imposición o la aceptación de préstamos. Este bilingüismo, en el grado en que se presente puede, con todo, constituirse en el punto de partida para una asimilación lingüistico-cultural a mediano o largo plazo, según, también la existencia de menor o mayor número de factores favorables. 


\section{CONCRECIONES EN RELACION AL ESTUDIO DE LA IDENTIDAD CULTURAL.}

De acuerdo a lo que hemos precisado a lo largo de esta exposición acerca de la relación entre lenguaje y cultura se pueden resumir, para los fines del proyecto sociológico sobre identidad cultural en la zona fronteriza,los enfoques principales en que el aspecto sociolingüístico deberá ser tomado en consideración. Partiremos de la afirmación de que hay una estrecha relación entre los dos conceptos, pero que esta relación es una de las tantas que se dan entre los hechos sociales y la cultura y de que, si bien el lenguaje la refleja, es sólo una de las manifestaciones necesarias para llegar a obtener una mundivisión. La identificación cultural de una comunidad o conjunto de ellas será factible sólo en la consideración de los diferentes sectores culturales y de la relación entre ellos, en tal forma que el examen de cada uno proporcione visiones parciales que, una vez integradas en un todo, puedan complementarse mutuamente.

Al considerar el lenguaje como uno de los elementos culturales que permitirán identificar a los grupos que integran la sociedad que vive en la zona fronteriza bajacaliforniana, precisaremos las modalidades que se consideran en el estudio y que se refieren exclusivamente a: 1) empleo de diversos sociolectos o sea la variedad sociodialectal, 2) grado imperante de bilingüismo en relación a los dominios familiar, laboral y comercial, y, 3) la afluencia de préstamos extranjeros procedentes del inglés, en los mismos dominios sociales mencionados.

Para cada una de estas modalidades sociolingüísticas se buscará su precisión en los diferentes estratos sociales que integran la sociedad global bajacaliforniana, y que corresponden a la estructuración socioeconómica y étnica, a la que se agrega la consideración del factor de ocupación secuencial de la faja fronteriza.

De acuerdo al triple eje de coordenadas (cultura de frontera, identidad cultural y conocimiento científico) que constituye el hilo conductor para el desarrollo de la investigación en uno de sus subproyectos sociológicos ${ }^{1}$ dedicaremos especial atención al aspecto del uso lingüístico por parte del núcleo ocupacional de quienes se dedican a la actividad científica, específicamente en su relación a la terminología científica y la captación de fuentes extranjeras, su aceptación o rechazo de tal terminología extranjera y la influencia que el probable empleo de términos científicos extranjeros ejerza en su lenguaje cotidiano. En este sentido podremos plantearnos como hipótesis de trabajo, la siguiente:

1 El otro subproyecto sociológico es el que se refiere a: "Menores de conducta antisocial y su identidad cultural en la zona fronteriza bajacaliforniana". 
Los hombres de ciencia, por la índole propia de su actividad, están más propensos al empleo de un mayor número de términos extranjeros para el ejercicio de su actividad; este hecho influirá en una mayor flexibilidad para el empleo más frecuente de un sociolecto, así como para la aceptación de un bilingüismo creciente en los restantes ámbitos sociales, y, consecuentemente, hacia una más abierta actitud de aceptación de patrones culturales no tradicionales y en un pérdida creciente de identidad nacional.

Además del estudio sobre el uso de sociolectos, bilingüismo y préstamos extranjeros entre los miembros del grupo de los hombres de ciencia, se intentará, en un a segunda etapa del proyecto sociológico, o en una primera si los recursos financieros así lo permiten, la realización de un análisis exploratorio del lenguaje que se emplea en las transmisiones de los macrodifusores. Se buscará localizar el grado de empleo de los préstamos y de los términos extranjeros, particularmente en las emisiones de las estaciones locales de radio y televisión. Esta posible parte de la investigación no pretende un análisis profundo, simplemente un primer acercamiento al problema, con lo cual más adelante se podrá contribuir a la interpretación y explicación del nivel de identidad cultural que prevalece en los miembros de los grupos sociales considerados en esta etapa del proyecto.

Para concluir el tratamiento del lenguaje como un elemento cultural, es necesario insistir en el hecho de que la cultura está integrada por diferentes sectores de los cuales el lenguaje integra sólo uno de ellos, y que todos en conjunto forman parte del mundo simbólico de la sociedad en estudio. Sin embargo, para los fines operativos de la investigación cada sector deberá ser considerado como una unidad en sí misma, cuyo estudio será preponderantemente sociolingüístico o semiológico de acuerdo a sus características particulares; pero que en todo caso deberán ser aprehendidas básicamente a través de la aproximación empírica. En el caso de los sectores en los que los símbolos sociales sean las unidades de análisis predominantes, el empleo del análisis semiológico constituirá un reto metodológico debido a que enfrenta al investigador con un campo disciplinario aún en desbroce en el cual el trabajo empírico y la elaboración de técnicas exploratorias constituyen etapas escaso o nulamente experimentadas en el ámbito cultural, tanto a nivel nacional como internacional. De aquí que la inclusión de los sectores simbólicos que permitan un acercamiento a la determinación o clasificación de los niveles de identidad cultural, representen una posibilidad de aportación teórica y metodológica para el avance de uno de los campos de estudio en que se unen dos ciencias de lo humano: la sociología y la semiología, la una en total dominio de su ámbito de estudio, la otra en desbroce de sus posibilidades. 


\section{EL LENGUAJE COMO ELEMENTO CULTURAL DE IDENTIDAD SOCIAL}

\section{BIBLIOGRAFIA}

FOUCAULT, Michel, Las Palabras y las Cosas. Siglo XXI, México 1978, p. 5.

HAUGEN, Einar, Algunos Problemas en Sociolingüistica en: La Sociolingüistica Actual, Edit. Oscar Uribe Villegas, Instituto de Investigaciones Sociales, UNAM, México, 1974, pp. 84, 85, 87, 93.

URE, Jean y Jeffrey Ellis: El Registro en la Lingüistica Descriptiva y en la Sociología Lingüistica en: La Sociolingüistica Actual, Edit. Oscar Uribe Villegas, Instituto de Investigaciones Sociales, UNAM, México, 1974 , p. 117.

URIBE VILLEGAS, Oscar, Sociolingüistica: una introducción a su estudio, Instituto de Investigaciones Sociales, UNAM, México, D. F., 1970, pp. 98, 109, 166. 